\title{
Effect of uncontrolled type-2 diabetes mellitus on the periodontal status of Saudi Arabian adults: A case-control study
}

Mir Faeq Ali Quadri ( $\nabla$ dr.faeq.quadri@gmail.com )

Jazan University Faculty of Dentistry https://orcid.org/0000-0001-6349-6136

Hammam Fageeh

Jazan University Faculty of Dentistry

Wael Ibraheem

Jazan University Faculty of Dentistry

Aadil Inamdar

Jazan University Faculty of Dentistry

Abbas Jessani

University of Saskatchewan College of Dentistry

Research article

Keywords: Diabetes, Periodontitis, Association, Risk factor, Case-Control study, Type 2 Diabetes, Saudi Arabia

Posted Date: April 30th, 2020

DOl: https://doi.org/10.21203/rs.3.rs-24049/v1

License: (c) (1) This work is licensed under a Creative Commons Attribution 4.0 International License. Read Full License 


\section{Abstract \\ Background}

The relationship between uncontrolled type-2 diabetes mellitus (T2DM) and periodontitis have been investigated among people from different nations. But, the current report is first from Saudi Arabia that utilized the gold standard measures to study the effect of uncontrolled T2DM on the periodontal status of ethnically similar Arab adults.

\section{Methods}

A case-control study was conducted, wherein cases were adults with clinically diagnosed periodontitis (clinical-attachment-loss $\geq 1 \mathrm{~mm}$ ). Concurrent controls were recruited from the same dental facility; matched with age, sex, and location. Uncontrolled T2DM was recorded using $\mathrm{HbA} 1 \mathrm{c}$ readings; and other health conditions including hypertension, epilepsy, bronchitis, thyroid disorders, and arthritis were retrieved from the participant's medical records. Data on tobacco consumption and related products (smoking, khat/qat, sheesha, shammah) was gathered using a self-perceived questionnaire. Frequencies, percentages, $p$-values, crude and adjusted odds ratios (OR) with 95\% confidence intervals were computed.

\section{Results}

Overall sample comprised of 166 cases and 332 controls with a mean age of 37.5 years. Multivariate analysis indicated uncontrolled T2DM as an important predictor for periodontitis among Saudi Arabian adults, and they had nearly three times greater odds (OR: $2.779 ; 95 \% \mathrm{Cl}: 1.425-5.419 ; \mathrm{p}=0.003$ ) of being diagnosed with periodontitis in contrast to non-diabetics. Secondary findings revealed that cigarette everusers were 2 times more likely to be suffering from periodontitis than never-users, and those brushing once per day or less had 5 times greater odds of developing periodontitis as compared to those brushing twice daily.

\section{Conclusion}

To conclude, the current case-control study is the first from Saudi Arabia to indicate that increased HbA1c level significantly effects periodontal status of Arab adults. An awareness of this association among health care providers and patients will contribute towards prevention of periodontitis in Saudi Arabia.

\section{Background}

Periodontitis is reported to have affected nearly $5-20 \%$ of the adult population worldwide [1, 2]. A common clinical sign of periodontitis is loss of dental connective tissue and bone, caused by inflammatory host response secondary to bacterial infection [3]. There are certain pathophysiological 
conditions that have the ability to disrupt the symbiotic equilibrium of microbes in the oral cavity, ultimately leading to periodontitis [4]. One such debilitating physiologic condition is diabetes, which is shown to be associated with periodontitis [5]. Among the two broad categories of diabetes, epidemiological studies have classified type-2 diabetes mellitus (T2DM) as a widespread condition, with current reports indicating approximately 285 million people with this disease, and estimating an increase of $50 \%$ by the year 2030 [6]. Reports indicate that blood sugar levels caused by diabetes may trigger a bacterial imbalance leading to a cascade of inflammatory reactions in the periodontal apparatus, causing loss of teeth, decreased chewing ability, nutritional imbalance, and eventually, a reduced oral healthrelated quality of life [7].

The certainty of association is agreed upon by many experts, and numerous studies and systematic analyses have been performed to demonstrate and evaluate the effect of diabetes on periodontitis. However, the findings of studies that report otherwise should also be considered. For instance, a systematic review that summarized findings through the year 2003 concluded that patients with diabetes had the same extent of periodontal disease compared with nondiabetics [8]. Furthermore, the results of a meta-analysis of four studies (9-12) did not show a significant difference between patients with and without diabetes in terms of clinical attachment loss (OR: $0.157 ; 95 \% \mathrm{Cl}:-0.034,0.347)$. Another systematic review, conducted in 2009, investigated the relationship between T2DM and periodontitis among 49 cross-sectional studies and 8 longitudinal studies. It concluded that most existing studies $(\mathrm{n}=$ 26) could have demonstrated better and precise findings if accounted for confounding variables, and had utilized robust statistical analyses and sample recruitment processes [9]. The most recent systematic review was performed in 2018 and revealed an increase in heterogeneity $\left(I^{2}=89.7 \%, p<0.001\right)$ among the included reports especially in terms of their study design [10], and the Center for Evidence Based Medicine (CEBM) specifies that proper study design and methodology and judgmental intuition of other potential factors are highly important to estimate an effect between the exposure and the outcome [11].

Till date there has been no evidence provided from an ethnically similar population of Saudi Arabian adults to substantiate the association of uncontrolled diabetes and periodontitis by utilizing the gold standard glycated hemoglobin $(\mathrm{HbA1c})$ test reports and clinical attachment loss, respectively. It is proclaimed that epidemiological studies are appropriate to investigate the association; among them cross-sectional studies exhibit limited causal inference as the exposure and outcomes are examined concurrently [12]. Cohort studies provide better evidence, but are not always feasible due to the huge enrollment required to demonstrate an effect, and the likely loss of follow-up data for some participants [13]. Due to these reasons, it is put forth that a case-control study design satisfies the criteria of temporality and is a more feasible approach in determining the association. With this, the current study aimed to investigate the association between uncontrolled T2DM (exposure) and periodontitis (outcome) among Saudi Arabian adult population; and it is hypothesized that the odds of developing periodontitis in participants with uncontrolled T2DM are higher than in their non-diabetic counterparts.

\section{Methods}




\section{Study location, study population, and study design}

The current study was conducted in Jazan province of Saudi Arabia, which is located in the southwestern part of the Arabian Peninsula, bordering Yemen. The site of data collection was the Dental Center affiliated with Jazan University. It is the largest oral health service provider in the region, catering to the needs of more than 1.5 million citizens, with the complete oral health cost borne by the Government of Saudi Arabia. The source population for this study was a cohort of 34,000 out-patients who visited the Dental Center between 2014 and 2017, of which 1,870 were diagnosed with periodontitis. An observational hospital-based case-control study was designed following the STROBE (Strengthening the Reporting of Observational Studies in Epidemiology) guidelines [14, 15].

\section{Sample size estimation}

In a previous nationwide study, the age-specific prevalence of T2DM among Saudi Arabian residents was reported to be $40.6 \%$, with Jazan region contributing to around $9.4 \%$ of the cases [16]. Due to lack of prevalence estimates of periodontitis in this region, the sample size calculation was based on data from the most recent available study performed in another region of Saudi Arabia [17]. This included $80 \%$ power, $95 \%$ precision, and an assumption of $0.5 \%$ of controls being exposed to the risk factor. The sample, was inclusive of two controls for each case (1:2). The estimated sample size required to reject the null hypothesis, with a $10 \%$ addition for dropouts was determined to be 66 cases and 132 controls.

\section{Selection criteria}

Saudi Arabian adults confirming their identity by displaying the national card were recruited as cases and controls for the current study. This was done to ensure similar ethnic backgrounds and cultural habits of study participants. Patients presenting with a past history of periodontitis (more than three months ago), or having physical and mental disabilities, or patients who did not provide signed consent, were excluded.

\section{Defining the cases and controls}

Cases were defined as clinically diagnosed periodontitis patients who underwent oral health examinations at the Dental Center affiliated with Jazan University by two calibrated specialists using the method prescribed by the American Academy of Periodontology (2014). Controls were adults selected from the same Dental Center, who had not been diagnosed with periodontitis, and were matched with cases on the basis of age, sex, and location.

\section{Assessment of outcome variable}

Assessment of outcome was conducted using the gold-standard classification system for periodontal diseases and conditions [18]. A Williams's periodontal probe (Hu-Friedy ${ }^{\circledR}$ PW6) was used to measure clinical parameters, including CAL and probing depths (PD). Six sites per tooth were assessed, and a diagnosis of periodontitis was made when subjects presented with at least two sites with a PD $\geq 4 \mathrm{~mm}$ and a CAL $\geq 1 \mathrm{~mm}$ (one on each tooth) [18].

\section{Assessment of exposure variables}


Medical reports were first manually checked to confirm the history of T2DM, based on the date of first diagnosis confirmed through glycated hemoglobin (HbA1c) tests. Then, patients with the current HbA1c level higher than $48.8 \mathrm{mmol} / \mathrm{mol}$ or $6.5 \%$ were defined as those having uncontrolled diabetes. A medical history of hypertension, epilepsy, bronchitis, thyroid disorders, and arthritis was confirmed from the participant's medical records, and responses were dichotomized (yes/no). Oral hygiene practice was recorded by asking the participants if they preferred brushing their teeth twice a day, once a day, or less than once a day. The response received was dichotomized, and "twice per day" was considered as a good oral hygiene practice, while the other options were considered as a fair/poor oral hygiene practice. Data on tobacco and related products (smoking, khat/qat, sheesha, and shammah) commonly used in the region were gathered through a patient-evaluation questionnaire, and responses were subsequently dichotomized to classify the patients as ever-users and never-users. Previous users of the mentioned products who had abstained for more than 5 years were also considered as never-users.

\section{Specialist calibration}

Periodontitis among the study participants was diagnosed independently by two American board-certified periodontists who were part of the current study. The first $10 \%$ of the recruited cases were cross-examined to assess the calibration. The inter-examiner reliability was calculated as Kappa (unweighted Kappa), and the result obtained ranged between 0.75 and 0.90 with an average of $0.84 \pm 0.03$ for the eight patients, achieving a satisfactory calibration agreement [19].

\section{Data collection process}

The focus of data collection process was temporality (exposure before the outcome). At first, perceived history on periodontitis was gathered for each patient by asking the respondents: "Have you been diagnosed by your dentist with gum diseases more than three months ago?" Responses were dichotomized as yes/no, and were cross-verified using the available electronic oral health records of the patients. The subjects who responded 'no' and verified were subjected to the aforementioned eligibility criteria, and then the selected patients were sent for clinical oral examinations in the Department of Periodontics. Out of the total cases of periodontitis $(n=1870)$, all the eligible cases $(n=166)$ were selected. Twice the number of controls $(n=332)$ were then matched (age, sex, and location) and recruited. Both cases and controls were subsequently assessed for uncontrolled T2DM and other exposure variables.

\section{Statistical analyses}

Relationship between periodontitis and T2DM, as well as other covariates, were first identified using the Chi-square or Fisher's exact test, as appropriate. The associations were explored further with bivariate and multivariate logistic regression analysis, after adjusting for confounders. Data were presented as frequencies, percentages, p-values, crude OR and adjusted OR; with their 95\% Cl. Data entry and statistical analyses were performed using the Statistical Package for the Social Sciences v.24 (SPSS, IBM Statistics). A p-value of $<0.05$ was considered as significant.

\section{Results}


The sample consisted of 166 cases and 332 controls (1:2) from the available pool of patients. The mean age of the study sample was 37.5 years $(S D=5.1)$. None were mentally or physically challenged or had gestational diabetes, renal disorders, HIV-infection, cancers, or kidney disorders. Estimates for hypotension, T1DM, pregnancy, epilepsy, thyroid, and shammah, could not be computed as these conditions were only present in either the cases or controls, and were even excluded from the multivariate models.

Findings from the relationship of periodontitis with general health conditions are reported in Table 1. T2DM, hypertension, diabetes, epilepsy, allergic conditions, asthma, and bronchitis were the reported general health conditions in the study sample. Among them, T2DM was more common in the cases and the controls. A significant relationship of T2DM and hypertension with periodontitis was observed. Hypertension was reported in $4.8 \%$ of the cases and in $0.6 \%$ of the controls ( $p=0.003)$, while T2DM was reported in $28.9 \%$ of the cases and $5.7 \%$ of the controls $(p<0.001)$ (Table 1$)$.

Table 1

General health conditions and their relationship with periodontitis $(N=498)$

\begin{tabular}{|lllll|}
\hline General Health conditions & $\begin{array}{l}\text { Cases }(\mathbf{N}=166) \\
\mathbf{n}(\%)\end{array}$ & $\begin{array}{l}\text { Controls }(\mathbf{N}=332) \\
\mathbf{n}(\%)\end{array}$ & $\begin{array}{l}\text { Total } \\
\mathbf{n}(\%)\end{array}$ & p-value \\
\hline T2DM & $48(28.9)$ & $19(5.7)$ & $67(13.4)$ & $<0.001^{\star}$ \\
\hline T1DM & $1(0.6)$ & $0(0)$ & $1(0.2)$ & $0.333^{\dagger}$ \\
\hline Hypertension & $8(4.8)$ & $2(0.6)$ & $10(2.0)$ & $0.003^{\star}{ }^{\dagger}$ \\
\hline Hypotension & $0(0)$ & $2(0.6)$ & $2(0.4)$ & $0.555^{\dagger}$ \\
\hline Asthma & $5(3.0)$ & $13(3.9)$ & $18(3.6)$ & $0.611^{\dagger}$ \\
\hline Pregnancy & $0(0)$ & $2(0.6)$ & $2(0.4)$ & $0.555^{\dagger}$ \\
\hline Epilepsy & $1(0.6)$ & $0(0)$ & $1(0.2)$ & $0.333^{\dagger}$ \\
\hline Arthritis & $3(1.8)$ & $5(2.7)$ & $8(1.6)$ & $1.000^{\dagger}$ \\
\hline Allergies & $1(0.6)$ & $9(2.7)$ & $10(2)$ & $0.176^{\dagger}$ \\
\hline Thyroid disorders & $1(0.6)$ & $0(0)$ & $1(0.2)$ & $0.333^{\dagger}$ \\
\hline Bronchitis & $1(0.6)$ & $3(0.9)$ & $4(0.8)$ & $1.000^{\dagger}$ \\
\hline *Statistically significant $(\mathrm{p}<0.05),+$ Fishers' test & & \\
\hline
\end{tabular}

Findings from the relationship of periodontitis with oral hygiene habits and substance use are reported in Table 2. Brushing once per day or less as opposed to twice daily was observed significantly more in 
cases $(39.1 \%)$ compared to controls $(11.4 \%)(p<0.001)$. Smoking $(p=0.006)$ and khat/qat $(p=0.002)$ use was also found to be significantly higher in patients with periodontitis (Table 2).

Table 2

Relationship of oral hygiene practice and substance use with periodontitis $(\mathrm{N}=498)$

\begin{tabular}{|c|c|c|c|c|}
\hline Habits & $\begin{array}{l}\text { Cases } \\
(\mathrm{N}=166) \\
\mathrm{n}(\%)\end{array}$ & $\begin{array}{l}\text { Controls }(\mathrm{N}=332) \\
\mathrm{n}(\%)\end{array}$ & $\begin{array}{l}\text { Total } \\
\text { n (\%) }\end{array}$ & $\mathrm{p}$-value \\
\hline Cigarette $^{a}$ & $27(16.2)$ & $27(8.1)$ & $54(10.8)$ & $0.006^{*}$ \\
\hline Khat/qat ${ }^{\mathrm{a}}$ & $20(12.0)$ & $15(4.5)$ & $35(7.0)$ & $0.002^{*}$ \\
\hline Sheesha ${ }^{a}$ & $1(0.6)$ & $1(0.3)$ & $2(0.4)$ & $1.000^{\dagger}$ \\
\hline Shammah $^{a}$ & $1(0.6)$ & $0(0)$ & $1(0.2)$ & $0.333^{\dagger}$ \\
\hline Brushing once/day or less $\$$ & $65(39.1)$ & $38(11.4)$ & 103(20.6) & $<0.001^{*}$ \\
\hline
\end{tabular}

The findings from the bivariate and multivariate logistic regression analyses are presented in Table 3 . The crude analysis identified T2DM and hypertension as significantly associated with periodontitis. Patients with T2DM and hypertension had six times (OR: 6.701; 95\% Cl: 3.783-11.872) and eight times (OR: 8.354; 95\% Cl: 2-39.799) higher odds, respectively, of being diagnosed with periodontitis. The multivariate analyses were adjusted for asthma, bronchitis, and sheesha use. Adjusted analysis revealed that T2DM patients had nearly three times greater odds (OR: $2.779 ; 95 \% \mathrm{Cl}: 1.425-5.419 ; \mathrm{p}=0.003$ ) of being diagnosed with periodontitis than their non-T2DM counterparts (Table 3 ). 
Table 3

Binary and multivariate logistic regression analysis demonstrating the effect various exposure variables on periodontal status $(\mathrm{N}=498)$

\begin{tabular}{|c|c|c|c|c|c|c|}
\hline \multirow[t]{2}{*}{ Risk factors } & \multicolumn{3}{|l|}{ Crude } & \multicolumn{3}{|c|}{ Adjusted $^{\dagger}$} \\
\hline & OR & $95 \% \mathrm{Cl}$ & p-value & OR & $95 \% \mathrm{Cl}$ & $\mathrm{p}$-value \\
\hline T2DM & 6.701 & $(3.783-11.872)$ & $<0.001^{*}$ & 2.779 & $(1.425-5.419)$ & $0.003^{*}$ \\
\hline Hypertension & 8.354 & $(2-39.799)$ & $0.003^{*}$ & 3.279 & $(0.507-21.191)$ & 0.212 \\
\hline Arthritis & 1.204 & $(0.284-5.099)$ & 1.000 & 0.459 & $(0.095-2.230)$ & 0.334 \\
\hline Allergies & 0.218 & $(0.027-1.731)$ & 0.176 & 0.268 & $(0.029-2.474)$ & 0.245 \\
\hline Cigarette & 2.194 & $(1.241-3.880)$ & $0.006^{*}$ & 2.088 & $(1.013-4.305)$ & $0.046^{*}$ \\
\hline Khat/qat & 2.895 & $(1.441-5.816)$ & $0.002^{*}$ & 2.206 & $(0.922-5.279)$ & 0.075 \\
\hline Brushing once/day & 4.979 & (3.144-7.885) & $0.000 *$ & 5.630 & $(3.514-9.021)$ & $<0.001^{*}$ \\
\hline
\end{tabular}

Cigarette smoking, khat use, and oral hygiene practice were significantly associated with periodontitis in the crude analysis. The adjusted analysis however revealed that only patients with the habit of cigarette smoking were two times (OR: $2.088 ; 95 \% \mathrm{Cl}: 1.013-4.305 ; \mathrm{p}=0.046$ ) more likely to have periodontitis. Khat use was not significantly related. Finally, patients brushing only once per day or less had a five times greater chance of developing periodontitis as compared to patients brushing twice daily (Table 3 ).

\section{Discussion}

Epidemiologic studies in Arab nations, including Saudi Arabia suggest a consistent rise in the T2DM cases that need immediate attention $[16,20]$. The Center for Disease Control (CDC) has reported that individuals diagnosed with diabetes are more likely to suffer from severe inflammation and inadequate white blood cell functions, which can subsequently lead to destruction of periodontal tissues [21]. However, to properly study the effect of diabetes on periodontitis, it was important to distinguish diabetic patients with uncontrolled blood sugar levels from those with controlled blood sugar levels. This is because the degree of glycemic control may determine the vulnerability of the immune defense mechanism, which ultimately leads to the damage of periodontal tissues. Further, there are reports that suggest that T2DM in particular, has the ability to remain undiagnosed for a long time as the affected patients retain their capacity to produce insulin, and the symptoms are usually missed [22]. Thus, goldstandard measures as oppose to subjective measures were required to confirm the glycemic control in the diabetic patients. 
Although, the relationship between diabetes mellitus and periodontitis is well studied, but the current study is first in Saudi Arabia to investigate the effect of uncontrolled T2DM on periodontal status in an ethnically similar Arab population using a case-control study design. After adjusting for other systemic diseases and substance abuse in an age, sex, and ethnicity-matched case-control study, it is observed that subjects with uncontrolled diabetes had nearly three times higher likelihood of developing periodontitis. A recent research in Saudi Arabia with a different study design aiming to compare the levels of Advance Glycation End-products in the gingival crevicular fluids of patients suffering from chronic periodontitis, with and without T2DM, supported the findings of the current study. However, the stated limitation was the inclusion of patients with controlled diabetes mellitus along with uncontrolled diabetes mellitus. In addition, one group of patients with uncontrolled diabetes were prescribed with medications and were still reported as having abnormal glycemic control, indicating that other risk factors or covariates that could have led to this uncontrolled state of blood sugar level were not considered [23].

A longitudinal study among the Gullah African Americans also showed that uncontrolled T2DM relates to a nearly two times higher magnitude of periodontal tissue destruction compared to their counterparts (controlled diabetics and non-diabetics) [24]. The results are further substantiated by other investigations from the United Kingdom [25], United States of America [26], India [27], and Spain [28]. A systematic review and meta-regression of longitudinal studies also confirms this association. However, these findings from meta-regression indicated a $25 \%$ variability between the published studies with respect to their sample size [10]. It further reiterated that some of the included studies did not have a representative sample [10], and evidence suggest that a small sample size often over estimates the association between the exposure and the outcome [29].

Secondary findings from the current study revealed that the patients who brushed their teeth once per day or less had a five times greater chance of developing periodontitis than patients who brushed twice per day. Affirming this, Han et al. (2017) suggested that maintaining a good personal oral hygiene through brushing, flossing, and professional oral prophylactic treatment is an important strategy to prevent gingival diseases, including periodontitis [30]. Similarly, other studies have also unequivocally stated that poor oral hygiene practices increase the risk of periodontitis by nearly fivefold, and that the risk substantially reduces with regular tooth brushing and dental visits [31].

The multivariate model in the current study also indicated that cigarette ever-users have nearly double the likelihood of being diagnosed with periodontitis than never-users. This association between cigarette smoking and periodontitis is well-established [32,33]. However, the use of khat (Catha edulis), which is reported to be responsible for periodontal diseases among a section of Arab populations living in the Southern border of the Arabian peninsula and Yemen [34], did not demonstrate a significant relationship with the incidence of periodontitis in the current study. We suggest that there may have been a collinear effect of cigarette use and khat (Catha edulis) use. In support of our finding, the latest systematic review conducted by Al-Maweri et al. in 2018 also reported that the available evidence suggesting the effect of khat on periodontal tissues is weak [35]. 
The strength of the current study lies in the use of gold-standard measures for the exposure (T2DM) and the outcome (periodontitis) variables. The sample consisted of ethnically similar Arab adults, in which cases and controls were further matched by age, sex, and location. The current report was also unique in relation to some of the earlier studies as it specifically considered patients with uncontrolled T2DM, as recommended by experts [36]. The data collection process of this study focussed towards temporality by confirming that the outcome among patients followed their exposure to risk factors. The study also prevented selection bias by recruiting all the eligible cases. Lastly, multivariate analysis with cluster effect of common risk factors was computed to obtain the findings after taking potential confounders into account.

However, the results should be interpreted carefully, as a case-control study is not the best approach to demonstrate a cause and effect relationship. While we acknowledge that a prospective cohort study design would be preferable for the demonstration of causal association, this was not advisable in the present scenario due to the lack of a sufficiently large sample of uncontrolled T2DM patients. Also, in a prospective study a concern of attrition bias could have arisen when the two groups (with and without uncontrolled T2DM) were subjected to the required physician consultations for their T2DM condition. Thereby, leading to a controlled state of blood sugar level. In addition, it is also agreed upon that the findings of the current study may not be completely generalizable as the study was based on data gathered from a single hospital. However, the study was performed at the largest Dental Center in the region that caters free oral health care to the residents.

Thus, the findings from this study endorses routine periodontal assessment for patients with diabetes in Saudi Arabia, especially those with a history of uncontrolled blood sugar levels. Additionally, as patients with T2DM are often not aware of their medical condition, oral health care providers are also advised to refer patients with diagnosed generalized periodontitis and without self-reported diabetes to general practitioners.

\section{Conclusion}

To conclude, the current case-control study is the first from Saudi Arabia to indicate that increased HbA1c level significantly effects periodontal status of Arab adults. An awareness of this association among health care providers and patients will contribute towards prevention of periodontitis in Saudi Arabia.

\section{Abbreviations}

T2DM

Type 2 diabetes mellitus.

$\mathrm{HbA1C}$

Hemoglobin A1C.

CAL

Clinical attachment loss. 
CDC

Center for Disease control.

CEBM

Center for Evidence Based Medicine.

\section{Declarations}

\section{Ethics approval and consent to participate}

This study was approved by the Research Unit (RU), College of Dentistry, Jazan University. Written and signed consent was obtained from each participant recruited in the study. The study abides by the Helsinki Declaration presented by the World Medical Association.

\section{Consent for publication}

Not applicable.

\section{Availability of data and materials}

The dataset of the current study will be made available upon request.

\section{Competing interests}

The authors do not have any competing interests to report with regards to the current research.

\section{Funding}

The current study is self-funded and we declare that no external funds from any organization/s has been received for performing the study or for the dissemination of the findings.

\section{Authors' contributions}

MFAQ and AJ had contributed towards the conception, design, data analysis, interpretation, drafting, and critical revision of the manuscript. Al contributed with data analysis and interpretation. HF and WI performed data acquisition, drafting, and then revised the manuscript. All authors have read and approved the submitted version of the manuscript.

\section{Acknowledgements}

The authors would like to acknowledge the study participants. We would also like to thank the Dean, Dr. Adbulwahab Alamir and the research unit at the College of Dentistry for their constant support during the current project. All authors gave their final approval and agree to be accountable for all aspects of the work. There is no conflict of interest to be declared and the study was not funded by any external source. 


\section{References}

[1] J. M. Albandar, "Epidemiology and risk factors of periodontal diseases," (in eng), Dent Clin North Am, vol. 49, no. 3, pp. 517-32, v-vi, Jul 2005, doi: 10.1016/j.cden.2005.03.003.

[2] B. A. Dye, "Global periodontal disease epidemiology," (in eng), Periodontol 2000, vol. 58, no. 1, pp. 10-25, Feb 2012, doi: 10.1111/j.1600-0757.2011.00413.x.

[3] H. Löe, "Periodontal disease. The sixth complication of diabetes mellitus," (in eng), Diabetes Care, vol. 16, no. 1, pp. 329-34, Jan 1993.

[4] M. Kilian et al., "The oral microbiome - an update for oral healthcare professionals," (in eng), Br Dent J, vol. 221, no. 10, pp. 657-666, Nov 2016, doi: 10.1038/sj.bdj.2016.865.

[5] K. Kampoo, R. Teanpaisan, R. G. Ledder, and A. J. McBain, "Oral bacterial communities in individuals with type 2 diabetes who live in southern Thailand," (in eng), App/ Environ Microbiol, vol. 80, no. 2, pp. 662-71, Jan 2014, doi: 10.1128/AEM.02821-13.

[6] M. N. Leite RS, Fernandes JK, Hermayer K, "Oral health and Type 2 Diabetes," Am J Med Sci, vol. 345, no. 4, pp. 271-273, 2013.

[7] D. G. Haag, K. G. Peres, M. Balasubramanian, and D. S. Brennan, "Oral Conditions and HealthRelated Quality of Life: A Systematic Review," (in eng), J Dent Res, vol. 96, no. 8, pp. 864-874, Jul 2017, doi: 10.1177/0022034517709737.

[8] Y. S. Khader, A. S. Dauod, S. S. El-Qaderi, A. Alkafajei, and W. Q. Batayha, "Periodontal status of diabetics compared with nondiabetics: a meta-analysis," (in eng), J Diabetes Complications, vol. 20, no. 1, pp. 59-68, 2006 Jan-Feb 2006, doi: 10.1016/j.jdiacomp.2005.05.006.

[9] N. G. Chávarry, M. V. Vettore, C. Sansone, and A. Sheiham, "The relationship between diabetes mellitus and destructive periodontal disease: a meta-analysis," (in eng), Oral Health Prev Dent, vol. 7, no. 2, pp. 107-27, 2009.

[10] G. G. Nascimento, F. R. M. Leite, P. Vestergaard, F. Scheutz, and R. López, "Does diabetes increase the risk of periodontitis? A systematic review and meta-regression analysis of longitudinal prospective studies," (in eng), Acta Diabetol, vol. 55, no. 7, pp. 653-667, Jul 2018, doi: 10.1007/s00592-018-1120-4.

[11] CEBM, "Center for Evidence Based Medicine; Study Designs," ed: Nuffield Department of Primary Care and Health Sciences, 2019.

[12] H. Checkoway, N. Pearce, and D. Kriebel, "Selecting appropriate study designs to address specific research questions in occupational epidemiology," (in eng), Occup Environ Med, vol. 64, no. 9, pp. 633-8, Sep 2007, doi: 10.1136/oem.2006.029967. 
[13] P. Sedgwick, "Bias in observational study designs: prospective cohort studies," (in eng), BMJ, vol. 349, p. g7731, Dec 2014, doi: 10.1136/bmj.g7731.

[14] M. Malta, L. O. Cardoso, F. I. Bastos, M. M. Magnanini, and C. M. Silva, "STROBE initiative: guidelines on reporting observational studies," (in eng), Rev Saude Publica, vol. 44, no. 3, pp. 559-65, Jun 2010.

[15] E. von Elm et al., "The Strengthening the Reporting of Observational Studies in Epidemiology (STROBE) Statement: guidelines for reporting observational studies," (in eng), Int J Surg, vol. 12, no. 12, pp. 1495-9, Dec 2014, doi: 10.1016/j.jijsu.2014.07.013.

[16] K. A.-M. Al-Rubeaan, H; Khoja, T; Ahmad, N; Al-Sharqawi, A; Siddiqui, K; AlNaqeb, D; Aburisheh, K; Youssef, A; Al-Batil, A; Al-Otaibi, M; and Al Ghamdi, A., "The Saudi Abnormal Glucose Metabolism and Diabetes Impact Study (SAUDI-DM)," Annals of Saudi Medicine, vol. 34, no. 6, pp. 465-475, 2014.

[17] A. K. Al-Sharabi, H. Shuga-Aldin, I. Ghandour, and N. N. Al-Hebshi, "Qat chewing as an independent risk factor for periodontitis: a cross-sectional study," (in eng), Int J Dent, vol. 2013, p. 317640, 2013, doi: $10.1155 / 2013 / 317640$.

[18] G. C. Armitage, "Development of a classification system for periodontal diseases and conditions," (in eng), Northwest Dent, vol. 79, no. 6, pp. 31-5, 2000 Nov-Dec 2000.

[19] J. R. Landis and G. G. Koch, "The measurement of observer agreement for categorical data," (in eng), Biometrics, vol. 33, no. 1, pp. 159-74, Mar 1977.

[20] A. Alotaibi, L. Perry, L. Gholizadeh, and A. Al-Ganmi, "Incidence and prevalence rates of diabetes mellitus in Saudi Arabia: An overview," (in eng), J Epidemiol Glob Health, vol. 7, no. 4, pp. 211-218, 12 2017, doi: 10.1016/j.jegh.2017.10.001.

[21] CDC, "Centre for Disease Control and Prevention; National Health Interview Survey (NHIS)-2007," ed, Accessed on 22 September, 2018.

[22] R. A. DeFronzo, "Insulin resistance: a multifaceted syndrome responsible for NIDDM, obesity, hypertension, dyslipidaemia and atherosclerosis," (in eng), Neth J Med, vol. 50, no. 5, pp. 191-7, May 1997.

[23] Z. Akram, F. Alqahtani, M. Alqahtani, A. A. Al-Kheraif, and F. Javed, "Levels of advanced glycation end products in gingival crevicular fluid of chronic periodontitis patients with and without type-2 diabetes mellitus," (in eng), J Periodontol, vol. 91, no. 3, pp. 396-402, Mar 2020, doi: 10.1002/JPER.19-0209.

[24] D. Bandyopadhyay, N. M. Marlow, J. K. Fernandes, and R. S. Leite, "Periodontal disease progression and glycaemic control among Gullah African Americans with type-2 diabetes," (in eng), J Clin Periodontol, vol. 37, no. 6, pp. 501-9, Jun 2010, doi: 10.1111/j.1600-051X.2010.01564.x. 
[25] E. Heidari, M. Andiappan, A. Banerjee, and J. T. Newton, "The oral health of individuals with dental phobia: a multivariate analysis of the Adult Dental Health Survey, 2009," (in eng), Br Dent J, vol. 222, no. 8, pp. 595-604, Apr 2017, doi: 10.1038/sj.bdj.2017.361.

[26] C. Tsai, C. Hayes, and G. W. Taylor, "Glycemic control of type 2 diabetes and severe periodontal disease in the US adult population," (in eng), Community Dent Oral Epidemiol, vol. 30, no. 3, pp. 182-92, Jun 2002, doi: 10.1034/j.1600-0528.2002.300304.x.

[27] P. K. Khanuja, S. C. Narula, R. Rajput, R. K. Sharma, and S. Tewari, "Association of periodontal disease with glycemic control in patients with type 2 diabetes in Indian population," (in eng), Front Med, vol. 11, no. 1, pp. 110-119, Mar 2017, doi: 10.1007/s11684-016-0484-5.

[28] M. Tapias Ledesma, C. Gallardo Pino, Á. Gil de Miguel, and V. Hernández Barrera, "[Relation between diabetes and periodontal disease in an Spanish adult population]," (in spa), Aten Primaria, vol. 48, no. 8, pp. 558-559, Oct 2016, doi: 10.1016/j.aprim.2015.11.007.

[29] Z. Zhang, X. Xu, and H. Ni, "Small studies may overestimate the effect sizes in critical care metaanalyses: a meta-epidemiological study," (in eng), Crit Care, vol. 17, no. 1, p. R2, Jan 2013, doi: $10.1186 /$ cc11919.

[30] K. Han and J. B. Park, "Association between oral health behavior and periodontal disease among Korean adults: The Korea national health and nutrition examination survey," (in eng), Medicine (Baltimore), vol. 96, no. 7, p. e6176, Feb 2017, doi: 10.1097/MD.0000000000006176.

[31] A. Lertpimonchai, S. Rattanasiri, S. Arj-Ong Vallibhakara, J. Attia, and A. Thakkinstian, "The association between oral hygiene and periodontitis: a systematic review and meta-analysis," (in eng), Int Dent J, vol. 67, no. 6, pp. 332-343, Dec 2017, doi: 10.1111/idj.12317.

[32] N. A. Chrysanthakopoulos, "Risk factors for the progression of periodontal disease in a Greek adult population," (in eng), J Investig Clin Dent, vol. 8, no. 2, May 2017, doi: 10.1111/jicd.12199.

[33] M. Bhat, K. Roberts-Thomson, and L. G. Do, "Clustering of risk indicators for periodontal disease: A population-based study," (in eng), Community Dent Health, vol. 32, no. 3, pp. 158-62, Sep 2015.

[34] A. I. Al-Kholani, "Influence of Khat Chewing on Periodontal Tissues and Oral Hygiene Status among Yemenis," (in eng), Dent Res J (Isfahan), vol. 7, no. 1, pp. 1-6, 2010.

[35] S. A. Al-Maweri, S. Warnakulasuriya, and A. Samran, "Khat (Catha edulis) and its oral health effects: An updated review," (in eng), J Investig Clin Dent, vol. 9, no. 1, Feb 2018, doi: 10.1111/jicd.12288.

[36] L. Casanova, F. J. Hughes, and P. M. Preshaw, "Diabetes and periodontal disease: a two-way relationship," (in eng), Br Dent J, vol. 217, no. 8, pp. 433-7, Oct 2014, doi: 10.1038/sj.bdj.2014.907. 